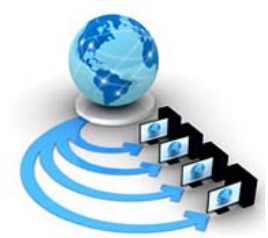

Volume 10, No. 3, May-June 2019

International Journal of Advanced Research in Computer Science

RESEARCH PAPER

\title{
Design and Implementation of a Residential Energy Management System
}

\author{
Ogunlere Samson ${ }^{1}$; Somefun Olawale ${ }^{2}$; Onilede Olutimi ${ }^{3}$; Mensah Yaw ${ }^{4}$ \\ 1, 2, 3,4 Department of Computer Science, School of Computing and Engineering Sciences, \\ Babcock University, Ilishan-Remo, Ogun State, Nigeria
}

\begin{abstract}
This paper proposes an optimization-based Residential Energy Management System (REMS) to minimize not only the customer's energy cost but also customer dissatisfaction with Power Holding Company of Nigeria (PHCN). REMS is designed in such a way that when there is a person in a particular room, electrical power is supplied to that room from its main source, and once the person leaves and the room, power supply is cut off, shutting off all appliances and devices in that room.This is achieved by the usage of a passive infrared sensor (PIR) to detect motion and an ArduinoMicrocontrollerATMEGA-328Pto process input data from the PIR sensors to determine if electrical power is needed in a room or not. AMicrocontroller PIC16F877Ais also usedto carry out all processing activities whose result would be effected through the aid of five output devices with a Liquid Crystal Display (LCD) used to display the PIR and the microprocessor status. The current state of the prototype design is described and a brief introduction to the control approach is made.
\end{abstract}

Keywords: Demand Response, Energy Management, Communication Protocols, Evolutionary Algorithms, Smart Grids.

\section{INTRODUCTION}

In recent years, technology has improved considerably. High-end systems used to direct the advancement of computer architectures and systems but now low-end systems drive technology due to their large volume and achievable profits.Developments in technology has therefore affected the way things are being done and has resulted in the rapid deployment of various power support systems and energy management systems over the years. The pace at which solutions are suggested and adopted have changed due to the readily-available technology that allows people to solve high - end problems quickly with ease. Presently, there are various metering platforms (electric billing system) for residents across the nation, but the methods of conserving energy are not adapting properly with these methods. Even in situations where residents complain of not supplied electric power or did not use up to what have been billed for, the bills keep on coming and the rates ever rising, resulting into what is known in Nigeria as 'crazy bill'. The traditional age-old system of power management and maintenance has failed to live up to the necessities of present times. Manual processes neither provide accurate, reliable or ample management decision support. It has resulted in lower power efficiency and utilization. The key to this ever-rising problem lies in the adoption of technology to create an effective and efficient energy management system that can work with the present electric power support system. An increased interest in electric demand response and the availability of smart utility meters have greatly increased the opportunities for residential energy management and enhanced their potential viability. To exploit these opportunities, a growing number of companies have emerged with various Residential Energy Management products and services over the past years, ranging from the fundamental energy displays to whole home control systems; termed, Home Energy Management Products \& Trends,(Janelle LaMarche, Katherine Cheney, Sheila Christian, and Kurt Roth, ND).
Energy management is the way to sparing vitality in the home. Quite a bit of its significance of vitality sparing frameworks emerged from the worldwide need to spare vitality.The worldwide need influences vitality costs, discharges targets and enactment, and the cost of vitality, all of which prompt a few convincing reasons why energy consumers ought to spare energy.Indeed, even at the High Level, the vitality office attempts to guard the lattice from digital and physical assaults; partners with states and different partners to arrange more safe foundation that can better withstand outrageous energy billings. Proficient energy use, is often essentially called energy efficiency; For instance, protecting a home permits a building to utilize less warming and cooling energy to accomplish and keep up an agreeable temperature. Introducing bright lights, LED lights or normal bay windows decreases the measure of vitality required to achieve a similar level of lighting contrasted and utilizing conventional radiant lights. Upgrades in energy proficiency are by and large accomplished by receiving a more proficient innovation or creation process or by utilization of regularly acknowledged techniques to decrease energy misfortunes, hence energy bulbs. There are numerous inspirations to enhance energy proficiency. Decreasing energy use diminishes energy costs and may bring about a money related cost sparing to consumers if the energy reserve funds counterbalance any extra expenses of executing an energy proficient innovation.

\subsection{Statement of Problem}

A large category of homeowners in Nigeria are not aware of the term, residential energy management system,because most residence take a more chaoticapproach to reducing energy consumption. The average residential building requires a significant amount of energy to run and remain comfortable; however, because humans are creatures of habit, it is often the case that such habits lead to significant energy losses and worse still, loss of lives and properties. For instance, the kitchen light may be left on when no-one is 
in the kitchen; the fans or air-conditioner units may be on when no-one is using them; the TV may be on when nobody is watching it. All these add up to high energy cost. With the daily increase in electric bills by power holding companies, some residents have resorted to unconventional illegal means of reducing power consumption by illegally tapping power from electric lines, thus short circuiting the system.These illegal connections often lead to lose of live, properties and prosecution with jail terms, all in the name of reducing the cost incurred when using electric power.The Residential Energy Management System proposed in this project is intended to give an answer for the issues experienced in the current energy utilization in Nigerian homes.Hence, a system like this is essential and highly recommended.

\subsection{Aim and Objectives}

The main aim of this project is to help manage energy in Nigerian homes.The objectives are to:

i. Identify the challenges faced by the residential energy management systems in Nigerian homes.

ii. Improve management on energy and costs savings by developing a more efficient residential energy management system that can maximize profit and minimize cost.

iii. Enhance the safety, security and comfort of energy consumersthat adopt residential energy management systems.

\subsection{Significance of Study}

The significance of this project is to examine the ways by which energy can be managed in a more effective and efficient ways with little or no human intervention /interference.

\section{REVIEWOF RELATED WORKS}

An occupant is by and large one who lives in a home. Energy management is the way to sparing energy in the home. A great part of the significance of energy sparing frameworks emerge from the worldwide need to save energy - this worldwide need influences energy costs which prompt a few convincing reasons why the inhabitant or occupant of a home ought to save energy.

\subsection{Energy and Environment}

According to market research reported by Rockwell Automation (2012), it would be troublesome for organizations today to be unmindful of energy use in their offices and homes. Energy expends an undeniably bigger share of working expenses, extricating, delivering to the end-users. Energy is fundamental for the production of riches and change of social welfare; this implies that sufficient and dependable supply of energy is required to guarantee maintainable advancement. Be that as it may, the utilization and transformation of essential energy more often than not brings about waste and discharge that are controlled from constrained assets which are considered naturally untenable. The expanding rate of ecological issues identified with energy utilization has prompted a developing enthusiasm for issues of reasonable advancement. It therefore, requires the clever utilization of assets, innovation, and strategic arrangement.

\subsection{Home Energy Management Systems}

Energy management signifies a huge open door for associations to decrease their energy utilize while keeping up or enhancing efficiency. The mechanical and business segments commonly represent around $60 \%$ of worldwide energy utilize. Associations in these areas can lessen their energy utilize $10 \%$ to $40 \%$ by successfully actuating anenergy management framework (EnMS). Deliberate energy management is a standout amongst the best ways to deal with enhancedenergy effectiveness in homes and businesses, since it outfits organizations with practices and systems to constantly make upgrades and catch new open doors.Energy management is a more extensive term, which applies diversely in various situations, while this project focuses on the one which is connected with energy sparing in homes, open segment/government associations or business. In this situation the way toward observing, controlling and saving energy in private and public building might be termed as energy management along these lines.

Energy management is characterized as the mindful utilization of energy to boost benefits (limit cost) and upgrade aggressive positions, in Guide to Energy Management by Cape hart, Turner and Kennedy, (1997). The most essential stride in the energy management process is the recognizable proof and examination of energy preservation openings, making it a specialized and administration work, to record, investigate, look at, modify and control energy through frameworks, so thatenergy is used with greatest effectiveness. Energy management system is any equipment as well as programming framework that can screen, control and save energy which requires a great deal of care and consideration.

\subsection{Chameleon Innovative Home Automation System}

Currently, few commercial products exist that automate an entire building for both convenience and power consumption. Commercially available automation system typically takes each system as an individual unit and typically assumes an unlimited power supply is available for each system. Energy management systems, on the other hand typically control to limit energy usage without regard to individual creature comfort. Research implementations such as 'Home for Life' perform similarly to Chameleon, but are designed for a fixed research implementation and lack the modularity required for mass adoption and confined refinement. Many building control systems are currently on the market. Siemens Control Logic of Michigan and Energy Control Systems Inc., for example, fall under the category of Energy Management Systems (EMS).

A project in Tokyo has set up a system that controls the airconditioner, using weather data provided from the internet, lights from occupancy and illumination sensors that provides energy usage information feedback to the resident for particular appliances. While the system provides comfort based on information and displays energy usage, it does not link the two together. Chameleon approach furthers this, by controlling a house utilizing this data to increase the efficiency. The Chameleon system contains world standard 
set of abilities, such as controls windows, recovers waste heat from the bathroom and controls appliances, (Bryan, 2010).

\subsubsection{Chameleon Capabilities}

Chameleon gets contribution from PCs, attractive card perusers, certain cell phones, embedded neighborhood touch screens and sensors. The framework speaks with gadgets for three particular purposes: accommodation, energy advancement and client instruction.

\subsection{Residential Energy Management System(REMS)}

A residential energy management system is a technology platform comprised of both hardware and software that allows the user to monitor energy usage and production and to manually control and/or automate the use of energy within a household.REMS is new to the market and have both advantages and in addition hindrances. These difficulties and advantages were examined at a Northeast Energy Efficiency Partnerships (NEEP) facilitated face to face workshop in July 15, 2015.

\subsubsection{Functionalities and Characteristics of} REMSProducts

To enable REMS technologies to meet their objectives, REMS technologies must offer a set of information and/or control based functionalities to users. These elements may include "residential utility demand response programs, home automation services, personal energy management, data analysis and visualization, auditing, and related security services” (Bojanczyk, 2013).

The main components of REMS include: sensing, monitoring and control devices; smart appliances; gateway devices; user interfaces and displays; and the enabling ICT (Bojanczyk, 2013; Azhar Fakharuddinet al., 2012). Despite this broad range of components, a single system does not require all to be present, resulting in a variety of REMS offering different benefits and demand management options. Although "REMS can be strictly software, strictly hardware, or a combination of both", a smart home that connects multiple devices may bring customer convenience and energy savings beyond what has been possible before. According to (Erol-Kantarci \& Mouftah, 2011; Strother \& Lockhart, 2013), past research suggests that effectiveness of REMS varies according to the type of system and its capabilities.

A review of academic and mainstream literature, coupled with a technology of REMSfall into three groups:

(1) User interfaces.

(2) Smart hardware.

(3) Software platforms as depicted in Table 1.

Table 1: REMS Category (Thayer, 2015)

\begin{tabular}{|c|c|c|}
\hline USER INTERFACE & SMART HARDWARE & SOFTWARE PLATFORM \\
\hline \multirow[t]{2}{*}{ Energy portal } & Smart Appliances & Smart Home Platform \\
\hline & Smart Thermostat & \\
\hline \multirow[t]{2}{*}{ Load monitor } & Smart Lightning & Data Analytics Platform \\
\hline & Smart plug & \\
\hline \multirow[t]{2}{*}{ In Home Display } & & Web Services Platform \\
\hline & Smart Hub & \\
\hline
\end{tabular}

\section{METHODOLOGY}

Residential Energy Management System serves the purpose of regulating the amount of electrical energy consumed in a home. Unlike the common energy management system which calculates the amount of electricity used by appliances in a home, this system is designed in such a way that when there is a person in a particular room, electrical power is supplied to that room from its main source, whereas, once the person leaves and the room is empty, power supply is cut off, shutting off all appliances and devices in that room. The methodological process adopted in this project is experimental and implementation.

\subsection{Design Procedure Specification and Consideration}

The project would make use of Passive Infra-Red sensors
(PIR) sensors as its input device to detect the presence of humans in the room. A PICMicrocontroller, PIC16F876A, would be used to carry out all processing activities whose result would be effected through the aid of five output devices.The project will take advantage of the processing ability of an ArduinoAtmega328pMicrocontroller known as LDR circuitto process input data from the PIR sensors to determine if electrical power is needed in a room or not. If the presence of a Human is detected in a room, signals would be sent to the power control center to supply electrical power to that room and the status indicator screen would display that the room is active, while other rooms without human presence would read inactive.Figure 1 shows the REMS design block diagram with various components and their roles in the system. 


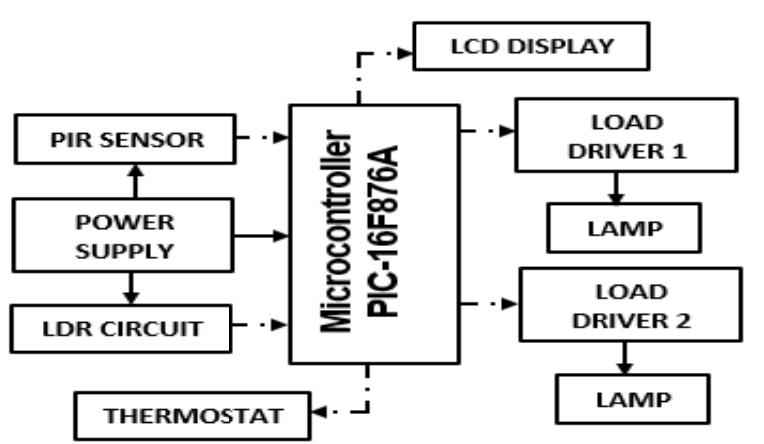

Figure 1: REMS Block Diagram

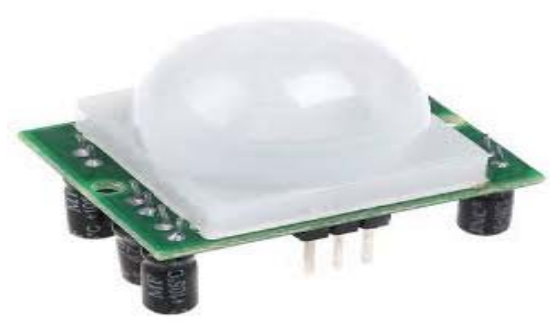

Fig.2: PIR Sensors,(adafruit)

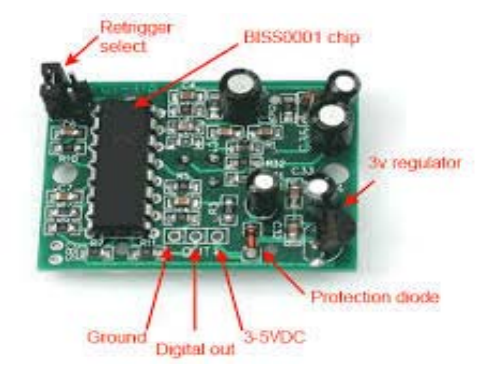

PIR (Passive Infra-Red sensors) are sufficiently sensitive to the presence of humans and have a range of up to 4 meters. They also come with semi-hemispherical plastic lenses which concentrate light from a wide angle for focusing on the sensor element; however, it was found that the lenses render the sensors too sensitive and so it was removed in this project.

\subsection{Description of Some Components Used}

\subsubsection{PIR Sensors}

\subsubsection{Arduino Uno}

An Arduino Uno was chosen for the micro-controller as it has adequate number of port pins (20) to drive the LCD status monitor, the Sensors and the actuator Switches. For this project, the number of pins required as depicted in Figure 4 are as follows:

1) 4 input pins for the sensors;

2) 6 output pins for the LCD;

3) 4 output pins for the Actuators

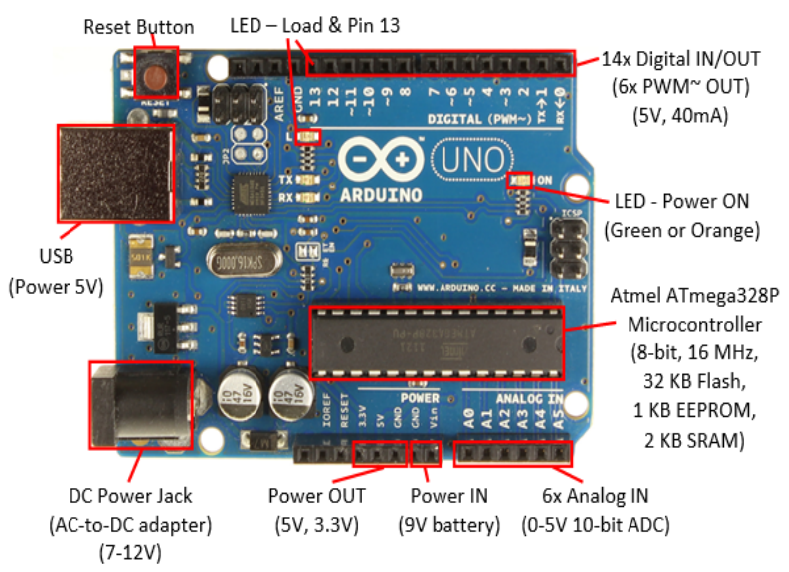

Fig.3: Arduino Uno Circuit

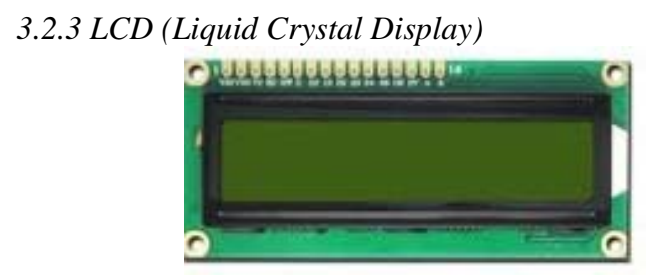

Fig.4: LCD Display

Without information on the current state of the system, it will be difficult to track down changes occurring in the system;hencean LCD display is included in the system to provide status information. This LCD runs in 4-bit mode to minimize hardware requirements.

\section{SYSTEM IMPLEMENTATION AND ANALYSIS}

\subsection{System Implementation}

Figure 5 shows the block diagram of the REMS project.

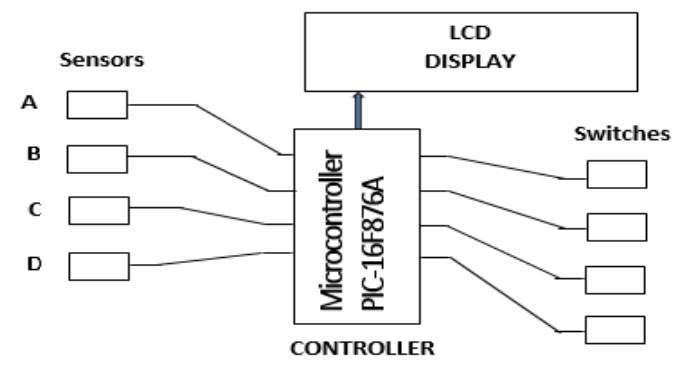

Fig. 5: Block Diagram of REMS

Figure 5shows the various modules tied together to form the complete system. To keep the diagram simple, the power supply is assumed but not shown. On the left are four 
potentiometers representing the PIR sensors; since they produce an output of 5 volts, they connect directly to the Analogue inputs of the Arduino Uno, ports A0-A3; the Arduino Uno's ADC are 10-bit units and so full range corresponds to 1024 which is 5 volts; in the code, it is specified that any measurement resulting $>500$ corresponds to logical 1 and anything below 500 to logical 0 .

The switches are NPN BC547/8 transistors, which require a few milliamps from the Arduino Uno to change state from OFF to ON. The requisite base drive current can be calculated thus:

Vo(Uno port output) $=5$ volts; Vbe(transistor base-emitter drop) $=0.6$ volts; Hfe(transistor DC currentgain $)=300(\mathrm{~min})$ see data-sheet; for a load current $\mathrm{I}(\mathrm{L})=160 \mathrm{~mA}$, $\mathrm{Ib}=\mathrm{I}(\mathrm{L}) / \mathrm{Hfe}=160 / 300=0.53 \mathrm{ma}$; now base resistance is then given by $\mathrm{R}=(\mathrm{Vo}-\mathrm{Vbe}) / \mathrm{Ib}=5-0.6 / 0.00053=4.4 / 0.00053=$ 8.3kiloohm(max);for worst case scenario, 5.6kiloohm was chosen; this allows for adequate margin even if the Arduino Uno's output for some reason does not get to 5volts. The value of load current used in the calculation is required by 3 white LEDs which represent domestic lighting in a domestic environment. The whole arrangement is powered by 4.5 volts (3xAA) size batteries. Since AA size batteries have an energy capacity of $300 \mathrm{maH}$,it will provide up to 30 minutes of simulated Domestic lighting:I(L) x4=160 x 4=640 mA; Duration of Service $=\mathrm{C}($ batt $) / \mathrm{I}(\mathrm{L})=0.3 / 0.64=0.469$ hours $=28$ minutes; (Hororwitz and Hill).

\subsection{Simulation of the Project with Software}

Proteus version 8.1 software was used to simulate the circuit; by representing the sensors with their appropriate counterparts available in Proteus, the operation of the system was stimulated even before the conclusion of fabrication. Based on such simulations, various modifications were made to the software.

\subsection{Physical System Implementation}

After simulating the designed circuit on Proteus and confirming it is working, the circuit design was built on a Veroboard and enclosed in a small model of a house (made of wood). The model was used to simulate a house with four rooms. The models have openings to access each room, so that hand can enter to represent the presence of a Human. Figures 6 and 7 showsthe developed model using wood to build a house.

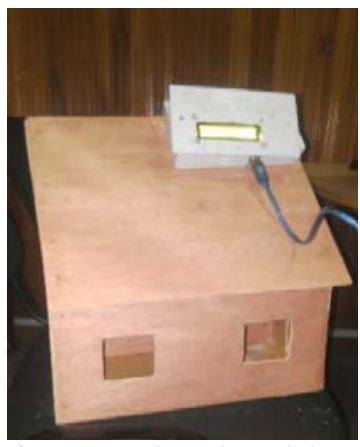

Fig 6: Developed model

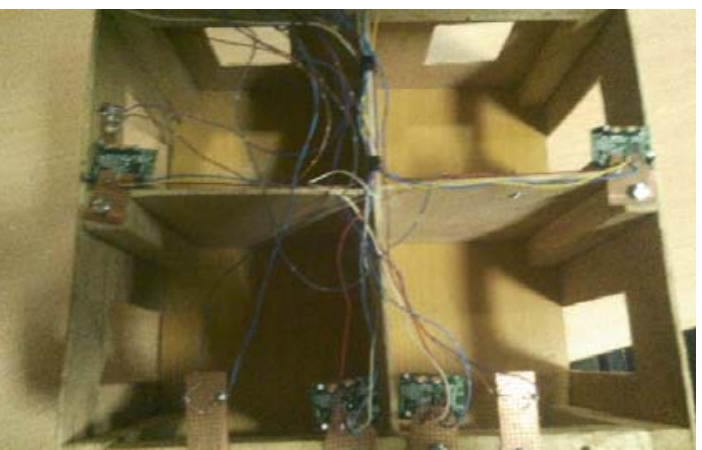

Fig 7: Inside the demo house

\subsection{Mode of Operation}

When the system boots up, the Micro-controller tests some of the lights and initiates the LCD screen; it then reads all the sensors to see if any are active, that is, if a human is present in the room; if any of the rooms are occupied, it activates the load switch to that particular room. As long as there is human presence within the room, it maintains the lights in that condition; meanwhile, it updates the LCD screen on the current status of the rooms. Once any of the rooms becomes vacant, unoccupied, it enters a time-out mode and at the expiration of the time, it disconnects power to the room.

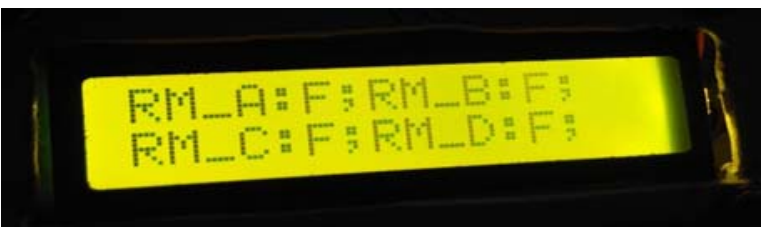

Fig 8: Status screen: RM_A representing room A, F representing free

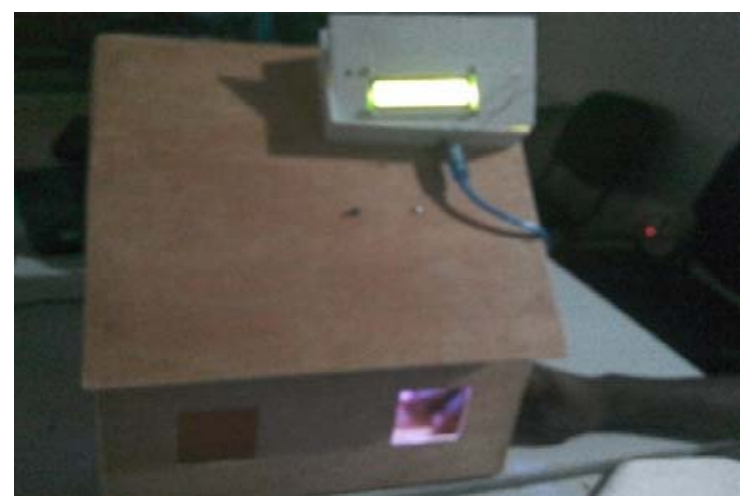

Fig 9: Live Testing done using a Hand to represent human presence in a room

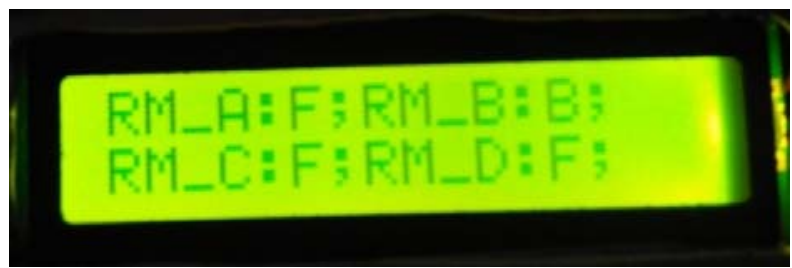

Fig 10: Status Screen indicating RM_B is Busy while RM_A, RM_C and RM_D are free. 
Figures 9 shows the live testing done using a hand to represent a human presence in a room $B$ which shows in the LCD of Figure 11 that a human is present in that room and thus activate all the electrical appliances in that only room. The indicators of Rooms A, C and D shows F (Free), an indication that no one is in those rooms.

Figure 11 shows the flow chart of REMS mode of operation.

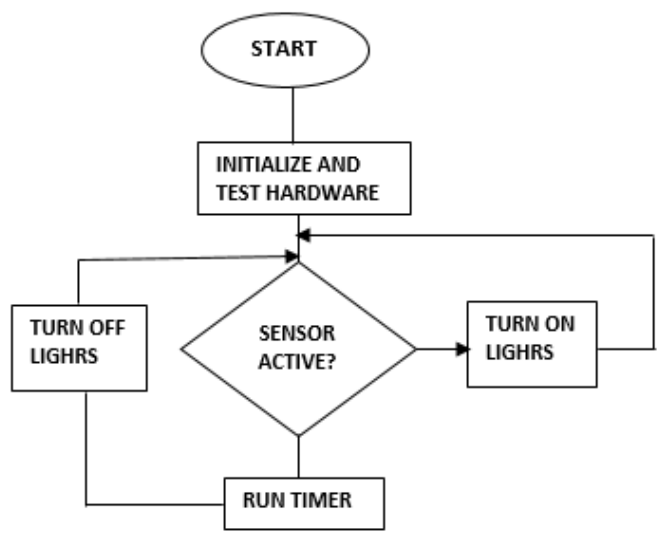

Fig. 11: Flow Chart of REMS mode of operations

\section{CONCLUSION}

The residential energy management system involves attention to detail in all processes. The purpose of this project is to provide a system that can be used by Nigerians to promote the culture of monitoring and maintaining energy used in the home. This study has contributed to knowledge by affirming that the residential energy management system has a significant impact on the use of energy within the home and this will however enhance the growth of the economy as a whole.

Further research can be carried using Ultra Sonic sensors to detect the presence of humans even when the human is in a fixed position.

\section{REFERENCES}

[1] Azhar Fakharuddin, Ahmad N. Abdalla, Muhammad
Rauf, Nik Mohd Kamil. (2012). A Smart energy management system for controlling and monitoring time of power consumption. Scientific Research and Essays, vol 7, pp. 1000-1011.

[2] Bojanczyk, K. (2013). Home Management Systems: vendors, technologies 2013-2017. Retrieved from Greentech media: hhtp://www.greentechmedia.com/research/report/homeenergy-management-systems-2013-2017

[3] Bojanczyk, K. (2013). Redefining home energy management system. Retrieved from Greentech media: http://www.greentechmedia.cim/articles/read/homeenergy-management-systemsredefined

[4] Bryan Glass, Ben Brannon, Katie Gratham, Staurt Baur. (2010). Expanding Horizons with Chameleon: Team Missouri's Innovative Home automation system. Energies ISSN 1996-1073, 1142-1153.

[5] Callaghan, P. (1992). Energy Management: A comprehensive guide to reducing costs by efficient energy use . London: McGraw Hill.

[6] Cape hart, Turner and Kennedy. (1997). Guide to Energy management 2nd Edition. Fairmont Press Inc.

[7] Erol-Kantarci and H. T. Mouftah, (2011), Wireless Sensor Networks for Cost-Efficient Residential Energy Management in the Smart Grid, IEEE ... 2.

[8] Janelle LaMarche, Katherine Cheney, Sheila Christian, Kurt Roth. (n.d.). Home Energy Management Products and Trends. Cambridge MA, USA: Fraunhofer.

[9] Kaur Harpreet \& Kamal deep Kaur,. (2012). An effective way of energy utilization. Energy conservation, vol 2, issue 5.

[10] Kim, J. (2016). Home Energy Management System (Base on the IoT Smart Home). Contemporary Engineering Sciences, vol 9, no 1, pp. 21-28.

[11]NEEP. (2015). Opportunities for Home Energy Management System (HEMS) in advancing residential energy efficiency program. NEEP.

[12] Report, M. R. (2012). Industrial Energy Management. Rockwell Automation.

[13] Roth K. and J. Brodrick. (n.d.). Home Energy Displays. ASHRAE Journal, Vol 50, no 7, pp. 136-137.

[14] Strother \& Lockhart, (2013), Category and functionality of Smart Home Technology for Energy Saving.

[15] Thayer, D. (2015). Characterization and potential of home energy management technology. Pacific Gas and Electric company. 